\title{
CircVAPA promotes proliferation and metastasis of retinoblastoma by modulating miR-615-3p and SMARCE1
}

Qibin Xu ( $\sim$ qibinxueyes@163.com )

\section{Primary research}

Keywords: circular RNA, retinoblastoma, circVAPA, miR-615-3p, SMARCE1

Posted Date: April 7th, 2020

DOI: https://doi.org/10.21203/rs.3.rs-20759/v1

License: (a) (i) This work is licensed under a Creative Commons Attribution 4.0 International License.

Read Full License 


\section{Abstract}

Background Growing evidence reveals that circular RNAs (circRNAs) play roles in cancer development. However, the effects and possible mechanisms of circRNAs in retinoblastoma (RB) are far from clear.

Methods circVAPA expression pattern was identified by RT-qPCR. CircVAPA induced effects on RB cells were tested by CCK-8, clone forming, flow cytometry and transwell assays. Bioinformatics assay, rescue experiments and dual luciferase tests were applied for mechanism exploration. Additionally, mouse models were established for in vivo assays.

Results circVAPA was expressed at high levels in human RB specimen and RB cell lines, and was correlated with poor outcomes of Rb patients. Knockdown of circVAPA could suppress the malignant phenotype of RB. Mechanistic experiments demonstrated that miR-615-3p could reverse the circVAPA induced effects on RB cells, and the downstream oncogene SMARCE1 was positively regulated by circVAPA via miR-615-3p. Further, in vivo analysis confirmed the findings.

Conclusions In summary, circVAPA promoted RB proliferation and metastasis by sponging miR-615-3p, thereby upregulating SMARCE1. CircVAPA is a potential biomarker of Rb therapy.

\section{Background}

Retinoblastoma (RB) is the most common malignant tumor in children under five years old [1]. RB is not sensitive to radiotherapy and chemotherapy. Although great efforts has been made to tackle this disease, the survival rate is under $5 \%$ in developing countries [2]. Hence, exploring biological mechanisms of RB progression and finding out biomarkers and therapeutic approach are urgent for diagnose and therapy of this disease.

Circular RNAs (circRNAs) are a subclass of non-coding RNAs with single-strands, and attracted great attention recently $[3,4]$. Most circRNAs are generated from exons with no protein-coding ability $[5,6]$. CircRNAs were reported predominantly located in cytoplasm, where they sponge miRNAs, thereby release the target genes sequestered by miRNAs [7, 8]. Emerging evidence has reported the important roles of circRNAs in diverse cancer types [9]. However, the function of circRNAs in RB has been rarely reported. CircVAPA, derived from vesicle-associated membrane protein-associated protein $A$, was a novel circular RNA that was recently found associated with cancer progression. For instance, Lin XN et al. found that circVAPA was upregulated and hampered colorectal cancer by sequestering miR-101 [10]. Liu C et al. was upregulated in hepatocellular cancer and enhanced cell proliferation [11]. Zhou SY et al. observed that circVAPA played as a tumor promoter via modulating miR-1615-5p in breast cancer development [12]. Nevertheless, the role of circVAPA in other caner types were not reported, including in RB.

In our work, we observed the overexpression of circVAPA in RB tissues, as well as the oncogenic effects on RB cells by promoting cell proliferation, migration and invasion. Further, the underlying molecular mechanism was explored and we revealed that circVAPA acted as an oncogene in RB progression via 
sponging miR-615-3p and thereby positively modulate SMARCE1. This research may help provide novel targets for RB clinical diagnosis and therapy.

\section{Methods}

\section{Clinical samples}

We obtained 50-paired RB tissue samples and adjacent normal ones from Zhejiang Medicine and Western Medicine integrated Hospital. The involved patients received surgery between 2017-2019. We obtained written informed consent from every participant, and this study was approved by the Ethics Committee of Zhejiang Medicine and Western Medicine integrated Hospital.

\section{Cell culture}

Human retinal pigment epithelial cell line (ARPE-19) and human RB cell lines (WERI Rb1, hTERT-RPE1, SORB-50 and Y79) were provided by Cell Bank of Type Culture of Chinese Academy of Sciences (Shanghai, China). Cells were subjected to RPMI-1640 medium (Gibco, USA) with 10\% FBS (Gibco, USA). Incubation was maintained at $37^{\circ} \mathrm{C}$ with $5 \% \mathrm{CO}_{2}$.

Real-time quantitative PCR (RT-qPCR)

Total RNA was extracted by Trizol (Invitrogen, USA). In each sample, $2 \mu \mathrm{g}$ RNA was used to synthesize cDNA as the templates of RT-qPCR. For, RT-qPCR,

\section{Cell transfection}

siRNAs against circVAPA (siVAPA), miR-615-3p overexpression plasmids (miR-615-3p mimics), miR-615$3 p$ inhibitor and corresponding negative controls were purchased from Integrated Biotech (Shanghai, China). Lipo3000 (Invitrogen, USA) was utilized for the subsequent transfection into cells.

\section{Cell viability assay}

CCK-8 Kit (Sigma, USA) was utilized to measure cell viability. Each well of a 96-well plate was seeded with 2000 cells and followed by an incubation of described time. Cells were then added with CCK-8 solution and absorbance was measured at $450 \mathrm{~nm}$.

\section{Colony forming assay}

Treated cells were seeded in 6-well plates with a density of 3000 cells per well, and cultured for 2 weeks. Then methanol was utilized for fixing and $0.5 \%$ crystal violet was applied for staining. After $30 \mathrm{~min}$, the number of colonies were counted.

\section{Cell apoptosis assay}

Cell apoptosis was detected using Annexin V/Cell apoptosis staining kit (LMAI Bio, Shanghai, China), according to the protocol. The cell apoptosis was tested using FACScan flow cytometer (BD Biosciences, 
San Jose, CA).

\section{Transwell assay}

Transwell chamber (Corning, USA) was utilized for migration and invasion assays. Cells were seeded in the upper inserts filled with RPMI-1640, and the lower chambers were filled with complete medium (10\% FBS). An incubation of 24-h later, the cells in the upper insets were removed. Cells in the lower chamber were fixed and stained with methanol and crystal violet. Migration cells were photographed using an inverted microscope (Olympus, Japan). For invasion assay, the upper chamber was pre-coated with Matrigel matrix (BD, USA).

\section{Western blot}

Cells or tissues were lysed and protein was extracted with RIPA (Thermo Fisher Scientific, USA). Equal amounts of each sample were loaded on 10\% SDS PAGE gel and transferred on PVDF membranes. Thereafter, $5 \%$ non-fat milk was utilized for blockage, and primary antibodies were used for incubation: SMARCE1 (Abcam, 1:1000) and beta-Actin (Tiangen, 1:2000).

\section{Immunohistochemical staining}

Tumors were treated with formalin and paraffin and sliced into $5 \mu \mathrm{M}$-thick sections. Xylene was utilized to deparaffinize the samples and ethanol was used for hydration. After blockage with serum for half an hour, samples were incubated with antibodies against SMARCE1 (1:200, Sigma) and Ki67 (1:500, Sigma) at $4{ }^{\circ} \mathrm{C}$ overnight. Secondary antibodies were taken for another incubation at room temperature for an hour. DBA was utilized for color reaction.

\section{Animal model}

Mice (8-week-old) were provided by Zhejiang Medicine and Western Medicine integrated Hospital. Y29 cells carrying sh-circVAPA or sh-scramble (control group) were injected into flanks of nude mice. Tumor volumes were recorded weekly. Four weeks later, mice were sacrificed and tumors were removed for the subsequent assays. Animal study was approved by Ethics Committee of Zhejiang Medicine and Western Medicine integrated Hospital.

\section{Results}

\section{CircVAPA was upregulated in RB and indicated poor prognosis}

To identify expression patterns of circVAPA, qPCR was applied. As shown in Fig. 1A-B, circVAPA expression levels was significantly increased both in RB patient tissue samples and cell lines. The characteristics of RB patients were listed in Table 1. Moreover, higher circVAPA expression indicated poorer prognosis (Fig. 1C). These findings suggested the potential oncogenic role of circVAPA. Thus, we used si-circVAPA for the subsequent functional experiments (Fig. 1D). 
Table 1

Characteristics of RB patients.

\begin{tabular}{|c|c|c|c|c|}
\hline \multirow{2}{*}{$\begin{array}{l}\text { Characteristics } \\
\text { Total }\end{array}$} & \multirow{2}{*}{$\begin{array}{l}N \\
50\end{array}$} & \multicolumn{2}{|c|}{ circVAPA expression } & \multirow[t]{2}{*}{$P$ value } \\
\hline & & High $(n=33)$ & Low $(n=17)$ & \\
\hline \multicolumn{5}{|l|}{ Age } \\
\hline$\geq 50$ & 35 & 25 & 10 & 0.521 \\
\hline$\otimes 50$ & 15 & 8 & 7 & \\
\hline \multicolumn{5}{|l|}{ Tumor size } \\
\hline$\geq 3$ & 28 & 18 & 10 & $0.032^{\star}$ \\
\hline$\nabla 3$ & 22 & 15 & 7 & \\
\hline \multicolumn{5}{|l|}{ FIGO stage } \\
\hline III-IV & 18 & 13 & 5 & $0.025^{\star}$ \\
\hline $\mid-I I$ & 27 & 20 & 7 & \\
\hline \multicolumn{5}{|c|}{ Lymph-node metastasis } \\
\hline Yes & 26 & 19 & 7 & $0.036^{*}$ \\
\hline No & 24 & 14 & 10 & \\
\hline \multicolumn{5}{|l|}{ Histological grade } \\
\hline Well & 20 & 15 & 5 & 0.418 \\
\hline Moderately/poorly & 30 & 18 & 12 & \\
\hline
\end{tabular}

Functional experiments were performed following si-circVAPA transfection. Cell viability was measured by CCK-8 assay, showing that silencing of circVAPA hampered cell viability in SO-RB-50 and Y79 cells (Fig. 2A). Colony forming experiments also showed the limited cell proliferation induced by circVAPA knockdown (Fig. 2B). Whereas, the apoptosis was aggravated by circVAPA silencing in SO-RB-50 and Y79 cells (Fig. 2C). As metastasis was an evident feature of cancer cells, we further tested the effects of circVAPA on RB cell metastasis. As shown in Fig. 2D-E, in circVAPA knockdown groups, the migration and invasion abilities were restrained markedly, compared with those in control groups. All these results confirmed the tumor promotional role of circVAPA in RB. 


\section{circVAPA served as a sponge for miR-615-3p}

We next explored the possible molecular mechanism. We utilized CircBank to predict miR-615-3p as an interacting miRNA, with potential binding sites presented in Fig. 3A. To confirm this prediction, we applied luciferase activity assay. As shown in Fig. 3B, luciferase activity was suppressed with co-transfection of miR-615-3p and wild type circVAPA, but not with co-transfection of miR-615-3p and mutant circVAPA. Moreover, miR-615-3p expression was deceased when circVAPA was knockdown (Fig. 3C). Further, we examined expressions of miR-615-3p in RB cell lines, observing the downregulated levels of miR-615-3p in SO-RB-50 and Y79 cell lines, compared with that in normal ARPE-19 cells (Fig. 3D). Also, miR-615-3p showed lower expression in RB tissue, in comparison with that in normal tissue (Fig. 3E). Additionally, there existed a negative correlation between circVAPA and miR-615-3p (Fig. 3F).

\section{circVAPA exerted oncogenic effects on RB cells via regulating miR-615-3p}

We further investigate whether miR-615-3p participated in circVAPA regulation on RB. Si-circVAPA was cotransfected with/without miR-615-3p inhibitor, followed by functional experiments. We observed that miR$615-3 p$ inhibitor attenuated the reduction of cell viability and colony number induced by si-circVAPA (Fig. 4A-B). The aggravation on cell apoptosis induced by si-circVAPA was also mitigated by miR-615-3p inhibitor (Fig. 4C). As for the status of cell metastasis, we observed that the downregulated migration and invasion abilities induced by si-circVAPA were both reversed upon miR-615-3p inhibitor tranafection (Fig. 4D-E). These observations indicated that miR-615-3p was involved in the regulation.

\section{circVAPA upregulated SMARCE1 via miR-615-3p}

We utilized TargetScan network tool to predict SMARCE1 as the potential target of miR-615-3p (Fig. 5A). We applied luciferase activity assay to confirm this prediction, As shown in Fig. 5B, luciferase activity was suppressed with co-transfection of miR-615-3p and wild type 3'UTR of SMARCE1, but not with cotransfection of miR-615-3p and mutant 3'UTR of SMARCE1. Moreover, SMARCE1 expression was deceased when miR-615-3p was overexpressed (Fig. 5C). These results confirmed that miR-615-3p directly interacted with SMARCE1. In addition, we observed that SMARCE1 was overexpressed in RB tissue, compared with that in normal tissue (Fig. 5D). Further, we found that SMARCE1 expression could be downregulated by circVAPA silencing both in mRNA and protein levels, and these alterations could be reversed by miR-615-3p (Fig. 5E-F). All the above observations indicated that circVAPA sponged miR-615$3 p$ to positively regulate SMARCE1 gene expression, which might be the underlying mechanism.

\section{circVAPA silencing hampered RB tumorigenesis in vivo}

Tumor xenografts were generated in mice to perform in vivo experiments. Y79 cells expressing shcircVAPA or sh-scramble (ctrl) were injected into mice. Tumor size and tumor weight were detected weekly and 4 weeks later respectively (Fig. 6A-B). After tumors were removed 4 weeks later, we examined the 
gene expressions in tumor tissues. RT-qPCR results showed that circVAPA and SMARCE1 were expressed higher in circVAPA depletion mice, while miR-615-3p was expressed lower (Fig. 6C-E). Immunohistochemistry assays were further performed to determine SMARCE1 and Ki67 expression levels. SMARCE1 was downregulated in sh-circVAPA mice, in line with the qPCR results we found (Fig. 6F). Ki67, a cell proliferation marker, was also lowly expressed in sh-circVAPA mice (Fig. 6G). The in vivo observations were consistent with those found in cell lines.

\section{Discussion}

$\mathrm{RB}$ is of high mortality rate that was commonly happened in children under five years old [13]. Despite of the great efforts in treating this disease, the survival rate is very low, especially in less developed countries [14]. Hence, it is urgent to find out new approach overcoming RB. Emerging evidence showed that circRNAs were dysregulated and exhibited tumor promotor or anti-cancer roles in caner progressions [15]. As to RB, there existed several reports demonstrating the relationship between circRNAs and RB development. For example, circ0001694 was revealed to overexpressed in human RB tissue and indicated poor survival rate by modulating AKT/mTOR signaling pathway [16]. Circ0006168 was reported to activate S6K/S6 signaling and modulating miR-384/RBBP7, thus contributing to RB cell viability and metastasis [17]. Thus, in this study, we aimed to investigate the influence of circRNA in RB pathogenesis.

CircVAPA, a newly discovered circRNA, was reported to play as a tumor promoter in several cancers. circVAPA was first found to play roles in colorectal cancer via interacting with miR-101 [10]. Recently, circVAPA was observed significantly upregulated in hepatocellular carcinoma and driven cancer development through sequestering miR-377-3p and modulating PSAP protein expression [11]. Moerover, circVAPA was also examined overexpressed in breast cancer and sponged miR-1615-5p [12]. However, whether circVAPA exhibited effects on RB pathogenesis remained unknown. In this study, we applied qRTPCR to determine the expression profile of circVAPA in RB. Results showed that circVAPA was highly expressed in RB tissue and cell lines. This result prompted us to explore the function of circVAPA in RB further. Next, loss-of-function assays were applied, and we demonstrated that knockdown of circVAPA could inhibit RB cell proliferation, migration and invasion, as well as aggravate cell apoptosis. These data were consistent with the tumor promoted role of circVAPA previously found.

Further, we aimed to explore the possible mechanisms. More and more reports confirmed that circRNAs likely to function by acting as sponges of miRNAs, thereby releasing the downstream target genes of miRNAs $[18,19]$. For example, BCRC-3 inhibited bladder cancer through sequestering miR-182-5p and positively regulating p27 [20]. Circ103869 downregulated miR-532-3p and released FOXO4 mRNA, thereby contributing to colorectal cancer cell proliferation [21]. Circ0084043 upregulated Snail via sponging miR153-3p to promote malignant melanoma [22]. In this study, we found that circVAPA could bind to miR615-3p, and the expression of miR-615-3p was lower in RB tissue and cell lines. Moreover, miR-615-3p could mitigate the effects on RB cells exerted by circVAPA. This suggested that miR-615-3p was involved in the regulation mechanisms. MicroRNAs are known to function by downregulating target genes [23]. Many miRNAs participated in cancer progression through repressing target mRNA expressions. For 
example, miR-376a-3p targeted KLF15 to promote colorectal cell proliferation and metastasis [24]. MiR498 promoted RB cell proliferation and inhibited cell apoptosis via targeting CCPG1 [25]. Notch 1 and PAX6 were suppressed to express normally by miR-433, thereby hampering RB progression [26]. Herein, we showed SMARCE1 as a direct target of miR-615-3p and found that SMARCE1 was highly expressed in RB tissue. SMARCE1 was a tumor promoter in many types of cancers, such as ovarian cancer [27], breast cancer [28], gastric cancer [29] and hepatoma carcinoma [30]. Additionally, we observed that the mRNA and protein expression levels of SMARCE1 were positively regulated by circVAPA via sponging miR-615, which might be the underlying mechanism.

\section{Conclusion}

CircVAPA exerted oncogenic effects on RB progression through sponging miR-615-3p and upregulating SMARCE1. This finding might provide new targets for clinical diagnosis and therapy of RB.

\section{Abbreviations}

RB

retinoblastoma

circRNA

circular RNA

\section{Declarations}

\section{Authors' contributions}

Qibin Xu designed the whole research, conducted the experiments and wrote the manuscript.

\section{Ethics approval and consent to participate}

The tissue samples were collected and were used in accordance with approval by the Ethics Committee of Zhejiang Medicine and Western Medicine integrated Hospital. All patients involved in this study were anonymous and provided written informed consent.

All animal experiments were approved by the Ethics Committee of Zhejiang Medicine and Western Medicine integrated Hospital, and all measures were undertaken to minimize the usage of animals as well as their suffering.

\section{Consent for publication}

Not applicable 


\section{Competing interests}

The authors declare that they have no competing interests.

\section{Availability of data and materials}

Not applicable

\section{Funding}

Not applicable

\section{Conflict of interest statement}

The author declares that there is no known competing financial interests or personal relationships that could have appeared to influence the work reported in this paper.

\section{References}

1. Dimaras H, et al. Retinoblastoma Lancet. 2012;379(9824):1436-46.

2. Singh G, Daniels AB. Disparities in Retinoblastoma Presentation, Treatment, and Outcomes in Developed and Less-Developed Countries. Semin Ophthalmol. 2016;31(4):310-6.

3. Meng S, et al. CircRNA: functions and properties of a novel potential biomarker for cancer. Mol Cancer. 2017;16(1):94.

4. Wu J, et al. Emerging Epigenetic Regulation of Circular RNAs in Human Cancer. Mol Ther Nucleic Acids. 2019;16:589-96.

5. Memczak S, et al. Circular RNAs are a large class of animal RNAs with regulatory potency. Nature. 2013;495(7441):333-8.

6. Chen LL, Yang L. Regulation of circRNA biogenesis. RNA Biol. 2015;12(4):381-8.

7. Szabo L, Salzman J. Detecting circular RNAs: bioinformatic and experimental challenges. Nat Rev Genet. 2016;17(11):679-92.

8. Glazar P, Papavasileiou P, Rajewsky N. circBase: a database for circular RNAs. RNA. 2014;20(11):1666-70. 
9. Hou LD, Zhang J. Circular RNAs: An emerging type of RNA in cancer. Int J Immunopathol Pharmacol. 2017;30(1):1-6.

10. Li XN, et al. Circular RNA circVAPA is up-regulated and exerts oncogenic properties by sponging miR101 in colorectal cancer. Biomed Pharmacother. 2019;112:108611.

11. Liu C, et al. Circular RNA circVAPA Promotes Cell Proliferation in Hepatocellular Carcinoma. Hum Gene Ther Clin Dev. 2019;30(4):152-9.

12. Zhou SY, et al., Circular RNA circVAPA regulates breast cancer cell migration and invasion via sponging miR-130a-5p. Epigenomics, 2020.

13. Benavente CA, Dyer MA. Genetics and epigenetics of human retinoblastoma. Annu Rev Pathol. 2015;10:547-62.

14. Ortiz MV, Dunkel IJ. Retinoblastoma J Child Neurol. 2016;31(2):227-36.

15. Vo JN, et al. The Landscape of Circular RNA in Cancer. Cell. 2019;176(4):869-81 e13.

16. Xing L, et al. Downregulation of circular RNA hsa_circ_0001649 indicates poor prognosis for retinoblastoma and regulates cell proliferation and apoptosis via AKT/mTOR signaling pathway. Biomed Pharmacother. 2018;105:326-33.

17. Xie ZF, et al. Circular RNA hsa_circ_0006168 contributes to cell proliferation, migration and invasion in esophageal cancer by regulating miR-384/RBBP7 axis via activation of S6K/S6 pathway. Eur Rev Med Pharmacol Sci. 2020;24(1):151-63.

18. Kulcheski FR, Christoff AP, Margis R. Circular RNAs are miRNA sponges and can be used as a new class of biomarker. J Biotechnol. 2016;238:42-51.

19. Zhang $X$, et al. Identification and comparison of novel circular RNAs with associated co-expression and competing endogenous RNA networks in pulmonary tuberculosis. Oncotarget. 2017;8(69):113571-82.

20. Xie F, et al., Circular RNA BCRC-3 suppresses bladder cancer proliferation through miR-182-5p/p27 axis. Mol Cancer, 2018. 17(1): p. 144.

21. Bian L, et al. Hsa_circRNA_103809 regulated the cell proliferation and migration in colorectal cancer via miR-532-3p / FOXO4 axis. Biochem Biophys Res Commun. 2018;505(2):346-52.

22. Luan W, et al. circRNA_0084043 promote malignant melanoma progression via miR-153-3p/Snail axis. Biochem Biophys Res Commun. 2018;502(1):22-9.

23. Mohr AM, Mott JL. Overview of microRNA biology. Semin Liver Dis. 2015;35(1):3-11.

24. Wang Y, et al., LncRNA TTN-AS1 sponges miR-376a-3p to promote colorectal cancer progression via upregulating KLF15. Life Sci, 2019: p. 116936.

25. Yang $L$, et al. miR- 498 promotes cell proliferation and inhibits cell apoptosis in retinoblastoma by directly targeting CCPG1. Childs Nerv Syst. 2018;34(3):417-22.

26. Li X, et al. MiR-433 inhibits retinoblastoma malignancy by suppressing Notch1 and PAX6 expression. Biomed Pharmacother. 2016;82:247-55. 
27. Giannakakis A, et al. Overexpression of SMARCE1 is associated with CD8 + T-cell infiltration in early stage ovarian cancer. Int J Biochem Cell Biol. 2014;53:389-98.

28. Sethuraman A, et al. SMARCE1 regulates metastatic potential of breast cancer cells through the HIF1A/PTK2 pathway. Breast Cancer Res. 2016;18(1):81.

29. Liu $\mathrm{H}$, et al. High expression of SMARCE1 predicts poor prognosis and promotes cell growth and metastasis in gastric cancer. Cancer Manag Res. 2019;11:3493-509.

30. Wu HJ, et al. miR-29a promotes hepatitis B virus replication and expression by targeting SMARCE1 in hepatoma carcinoma. World J Gastroenterol. 2017;23(25):4569-78.

\section{Figures}




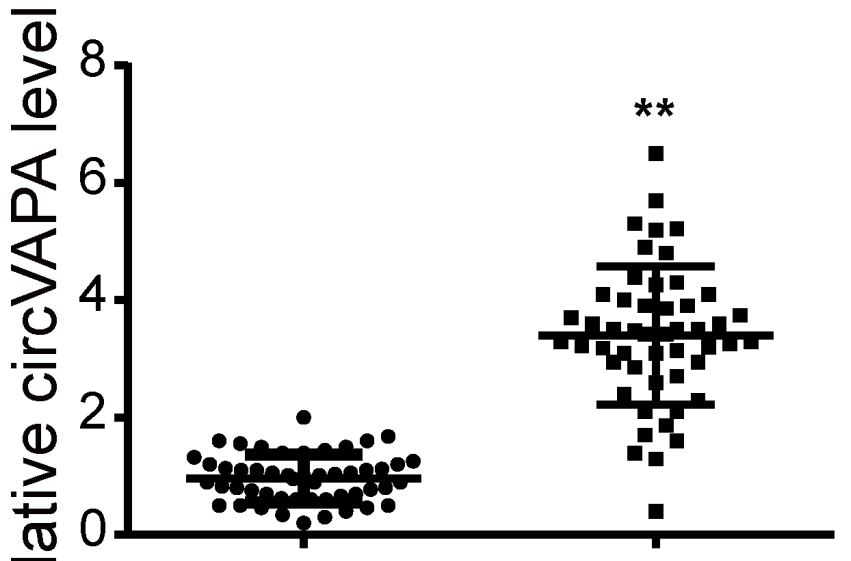

$\underset{\mathbb{\Phi}}{\mathscr{N}}$ Normal tissue RB tissue

C

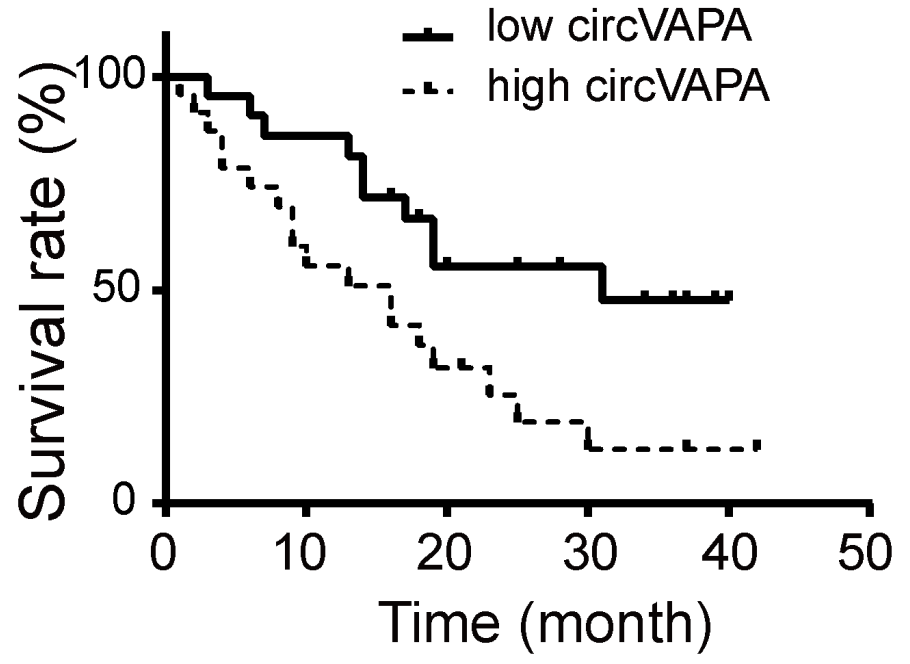

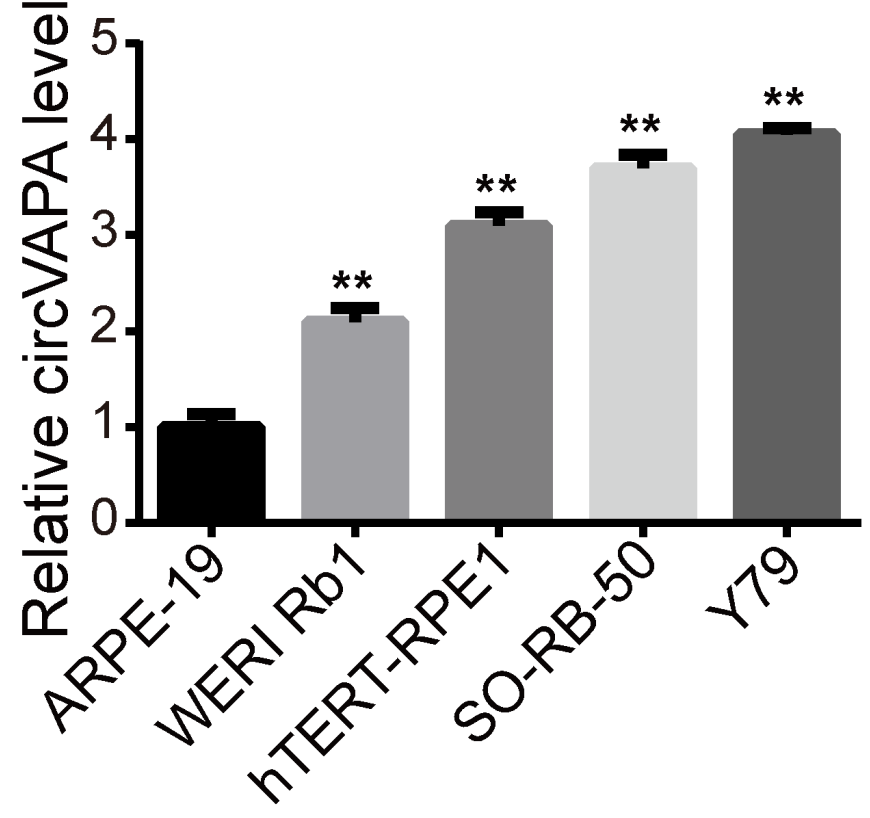

D

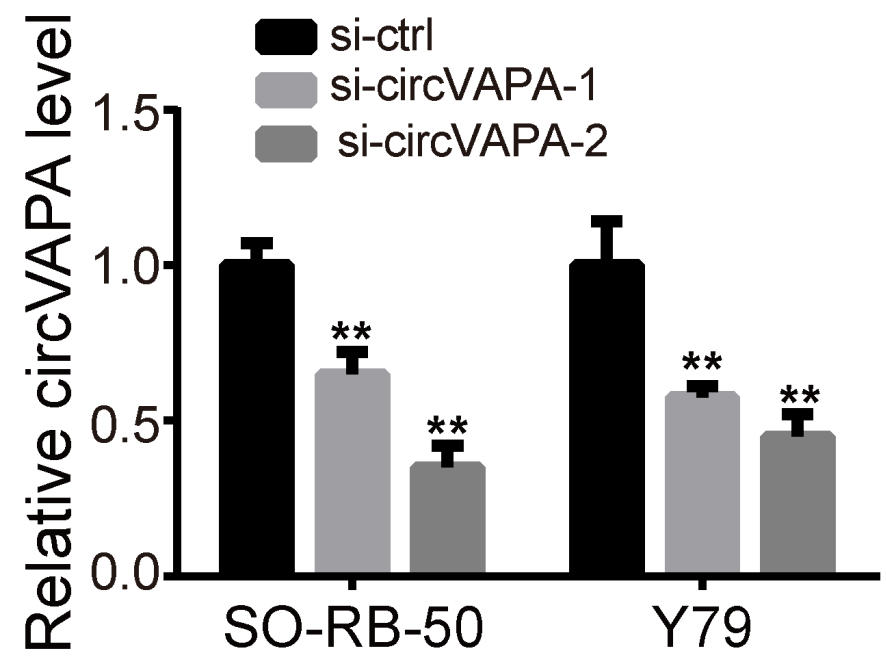

Figure 1

Expression level of circVAPA in RB. (A) circVAPA expressions in RB tissues and matched nearby normal ones were detected by qRT-PCR. (B) circVAPA expression levels in RB cell lines (WERI Rb1, hTERT-RPE1, SO-RB-50 and Y79) and human retinal pigment epithelial cell line (ARPE-19) were examined by qRT-PCR. (C) Kaplan-Meier survival analysis was utilized to analyze the association of circVAPA expression and overall survival. (D) siRNAs against circVAPA were transfected into RB cell lines, and the knockdown efficiencies were tested by qRT-PCR. ${ }^{*} \mathrm{P}<0.01$. 
A
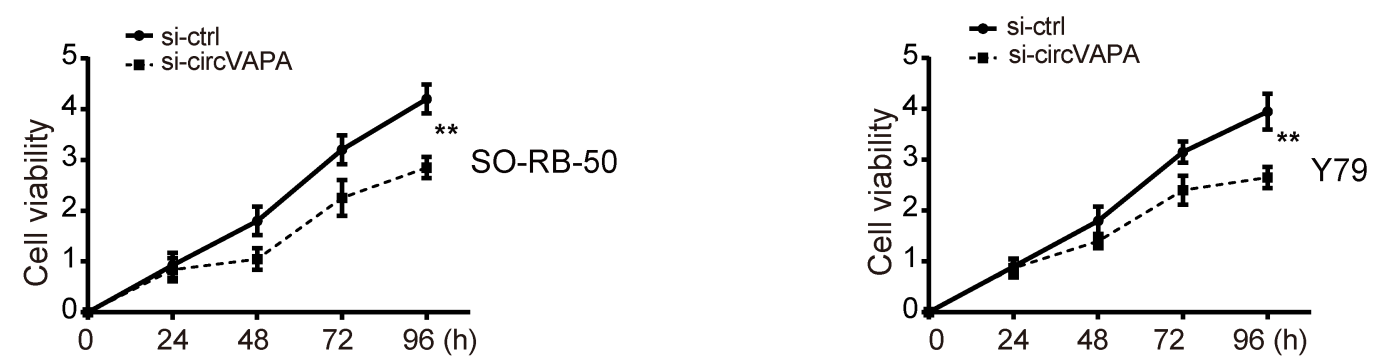

B

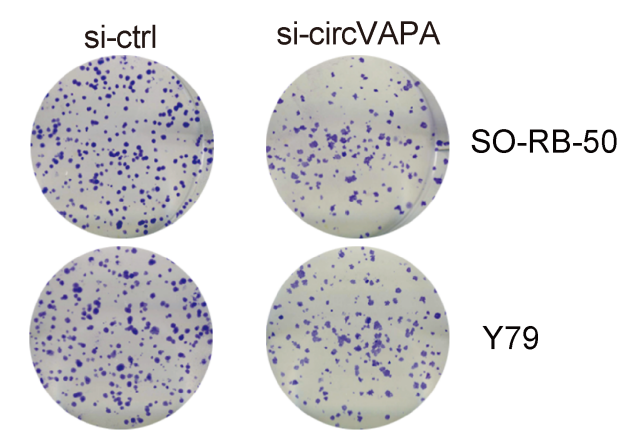

C
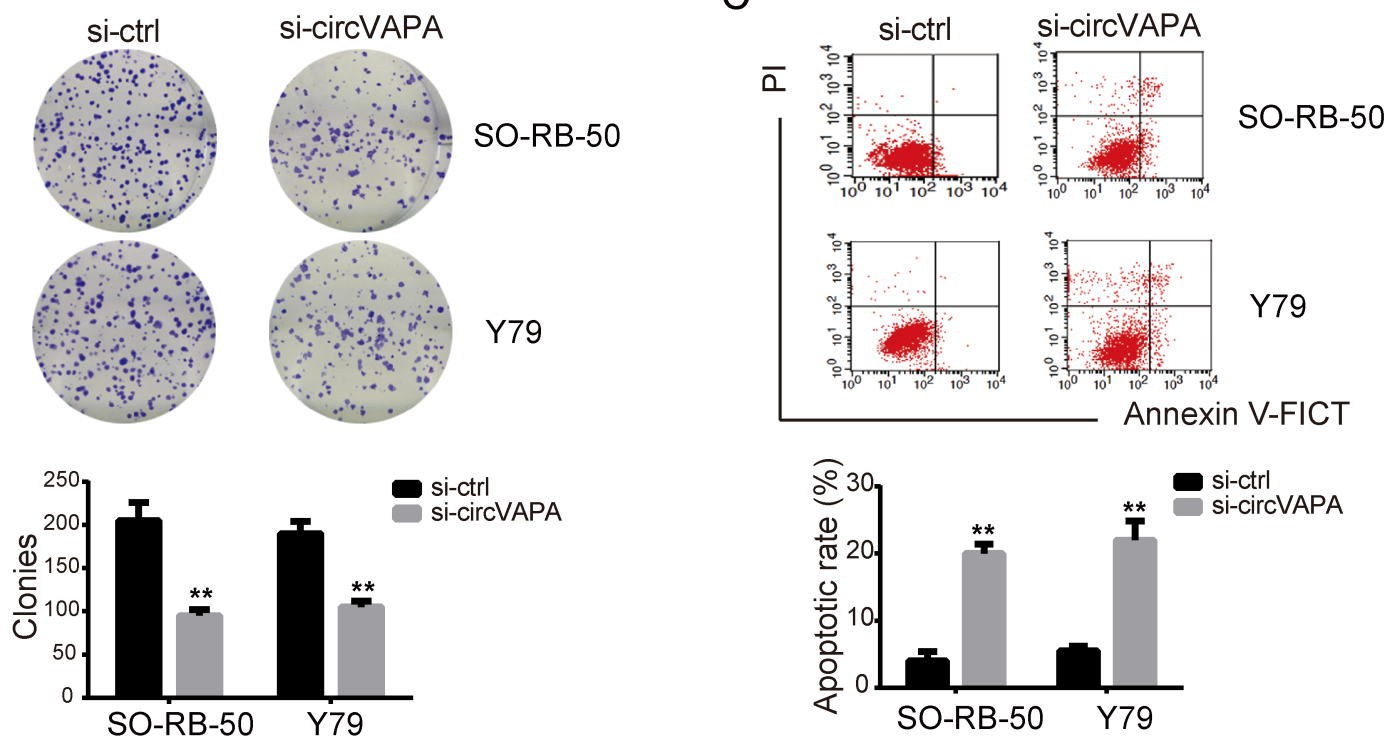

D

E
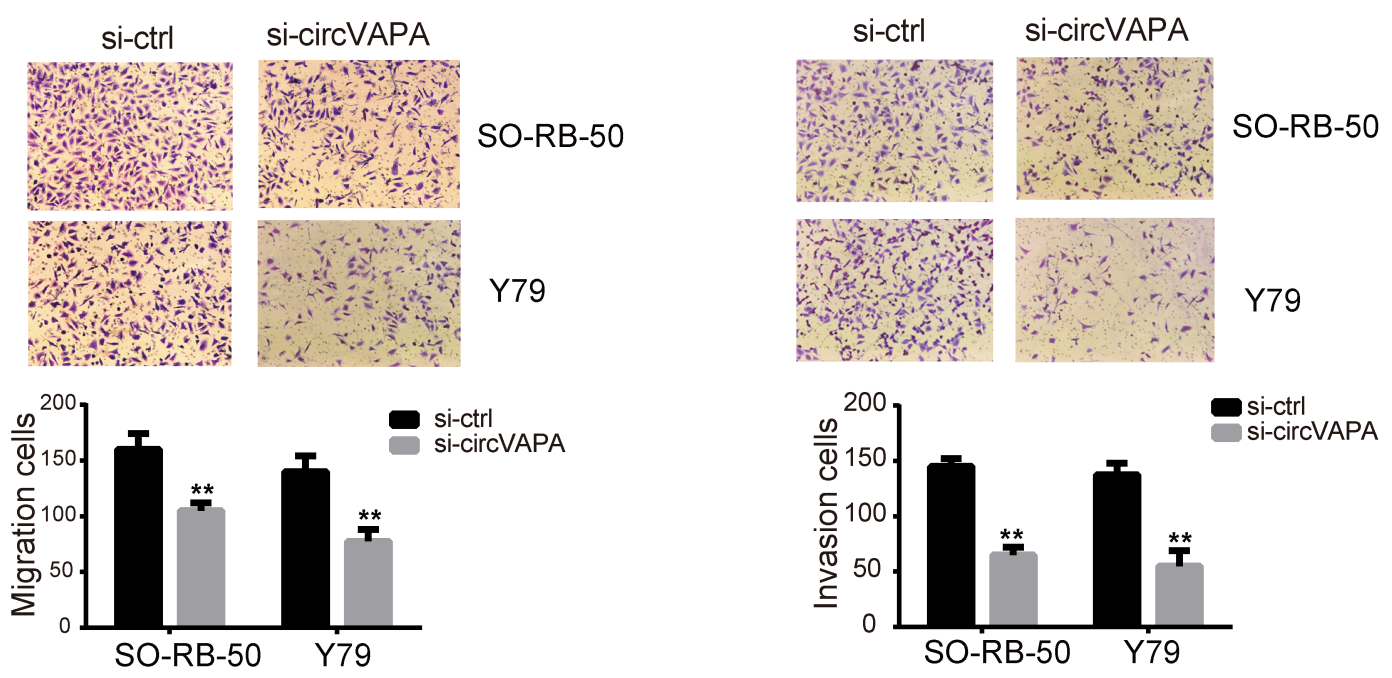

Figure 2

circVAPA exerted oncogenic effects on RB cells. Si-VAPA was transfected into SO-RB-50 and Y79 cells, (A) CCK8-assay was applied to assess cell viability; $(B)$ colony forming experiments were performed to evaluate cell proliferation; (C) Flow cytometry was carried out to test cell apoptpsis; (D) trasnwell assays were utilized to detect cell migration; $(\mathrm{E})$ trasnwell assays were used to determine cell invasion. ${ }^{* \star P}<0.01$. 
A
circVAPA (wt) 5'-...CGGCAGGCGTTAGGGCTCGGG...-3'
miR-615-3p 3'-UUCUCCCUCUGGGUCCGAGCCU -5'
circVAPA (mut) 5'-...CGGCAGGCGTTAGUUUAACCG...-3'

$\mathrm{B}$
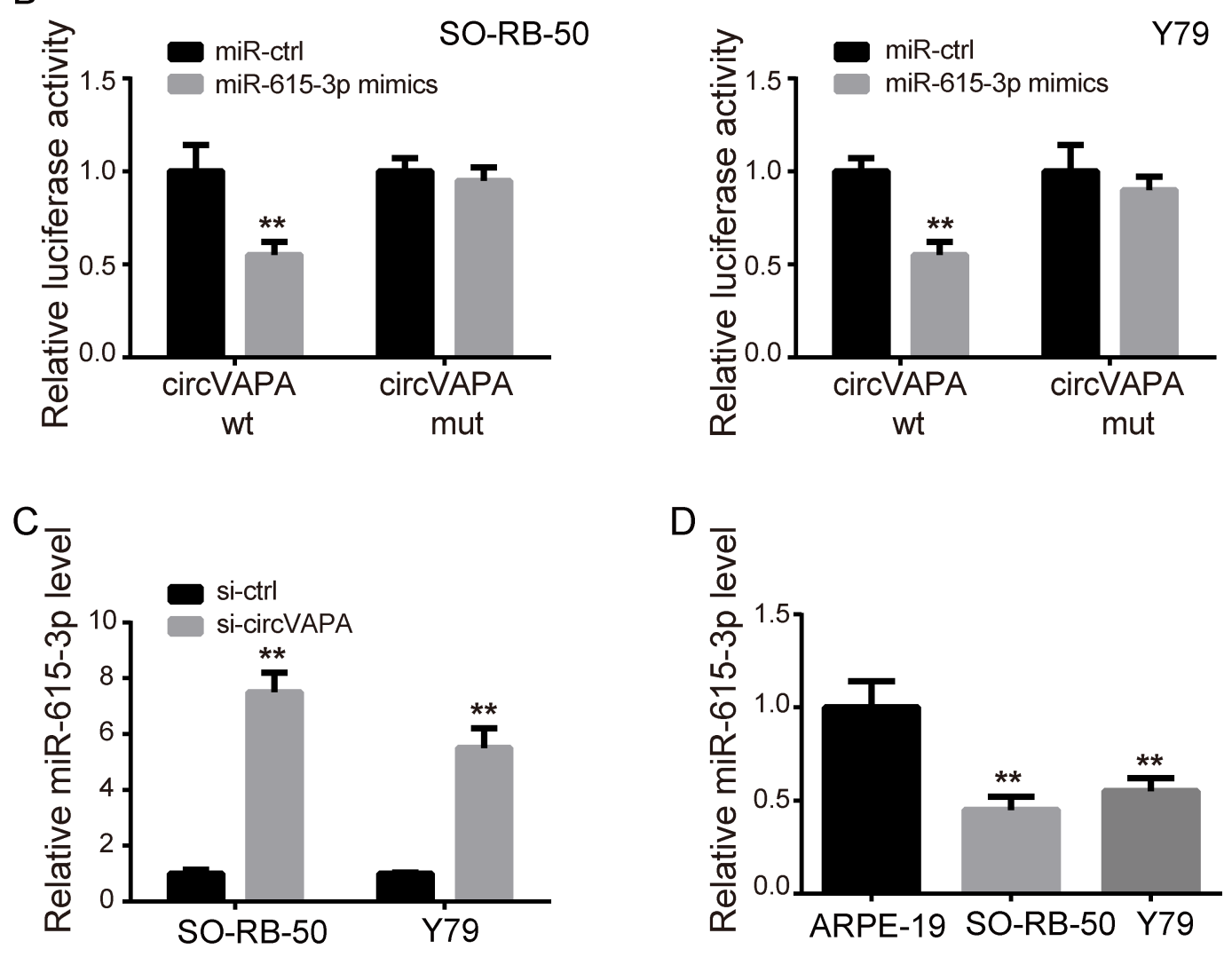

$\mathrm{D} \frac{\bar{\Phi}}{\underline{\mathrm{d}}}$
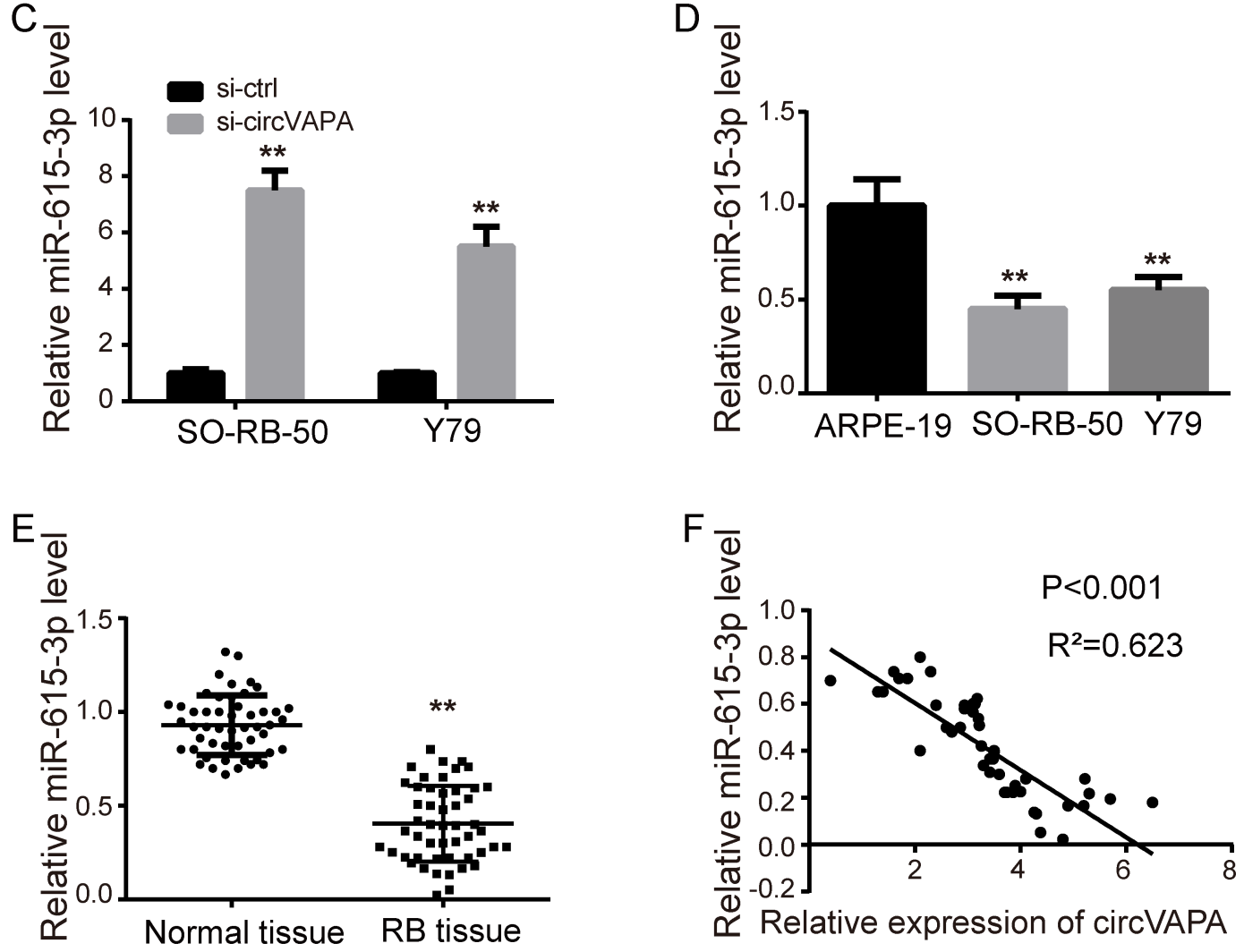

\section{Figure 3}

circVAPA was a sponge for miR-615-3p. (A) Putative interacting sites predicted by CircBank. (B) Luciferase activities were detected after co-transfection of miR-615-3p and circVAPA wt/mut. (C) sicircVAPA was transfected, and miR-615-3p expression was measured by qRT-PCR. (D) miR-615-3p expression levels in RB cell lines (SO-RB-50 and Y79) and normal cell line (ARPE-19) were measured by RT-qPCR. (E) miR-615-3p expression levels in RB tissues and matched nearby normal ones were 
measured by RT-qPCR. (F) Pearson's correlation analysis of circVAPA and miR-615-3p in RB tissue. **P $<0.01$.

A

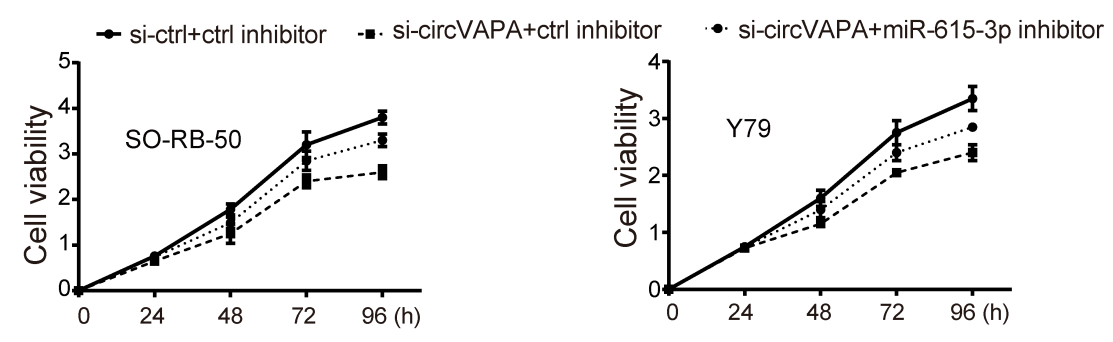

B

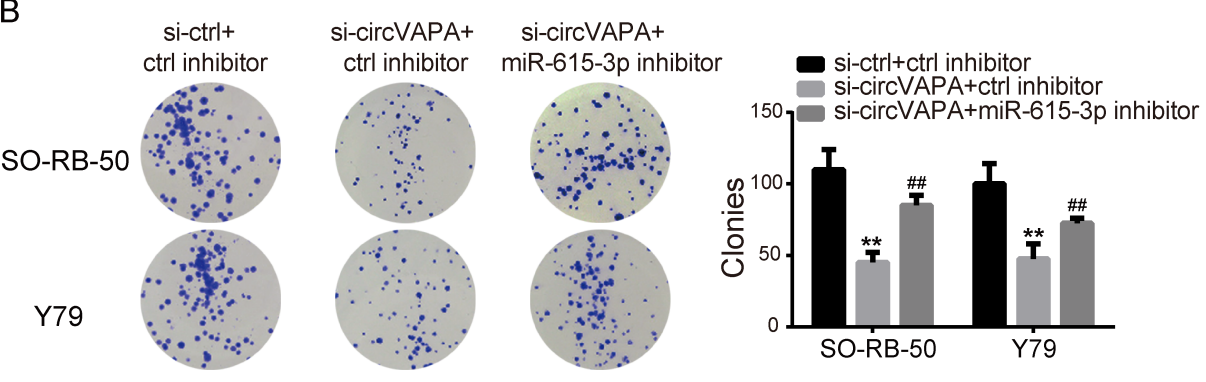

C si-ctrl+ $\begin{array}{ccc}\text { si-circVAPA+ } & \text { si-circVAPA+ } \\ \text { ctrl inhibitor } & \text { mibitor } & \text { miR-615-3p inhibito }\end{array}$

SO-RB-50

$\overline{0}$ :
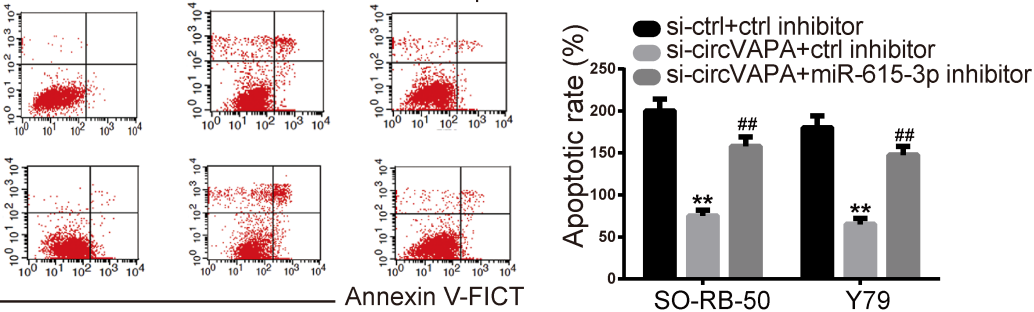

D
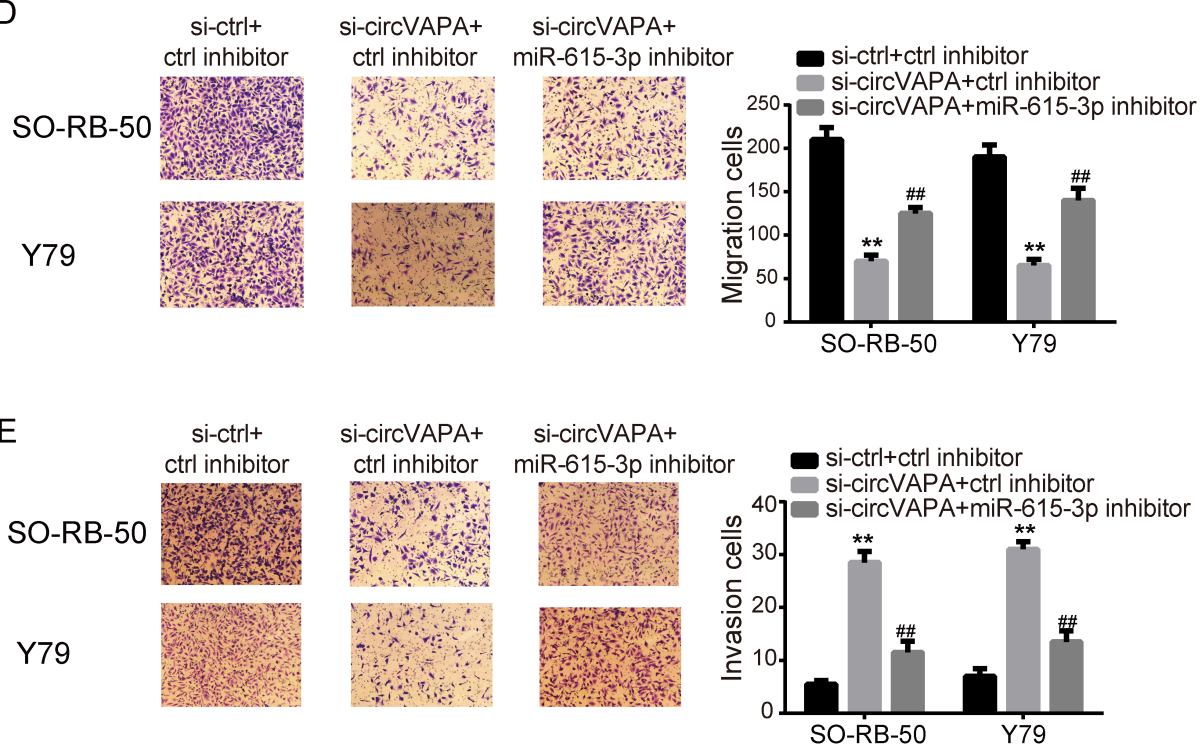

Figure 4

miR-615-3p inhibitor mitigated the si-circVAPA induced-oncogenic effects on RB cells. Si-circVAPA was cotransfected with miR-615-3p inhibitor or its negative control inhibitor, (A) CCK8-assay was applied to assess cell viability; (B) colony forming experiments were performed to evaluate cell proliferation; (C) 
Flow cytometry was carried out to test cell apoptosis; (D) trasnwell assays were utilized to detect cell migration; (E) trasnwell assays were used to determine cell invasion. ${ }^{*} \mathrm{P}<0.01$.

A

SMARCE1 wt $\quad 5 '$ '...AGUGAUAGUAACACUGGCUCGGA...

| || | | | |

miR-615-3p 3' UUCUCCCUCUGGGUCCGAGCCU

SMARCE1 mut $\quad 5^{\prime}$...AGUGAUAGUAACACUCCAAACCA...

B
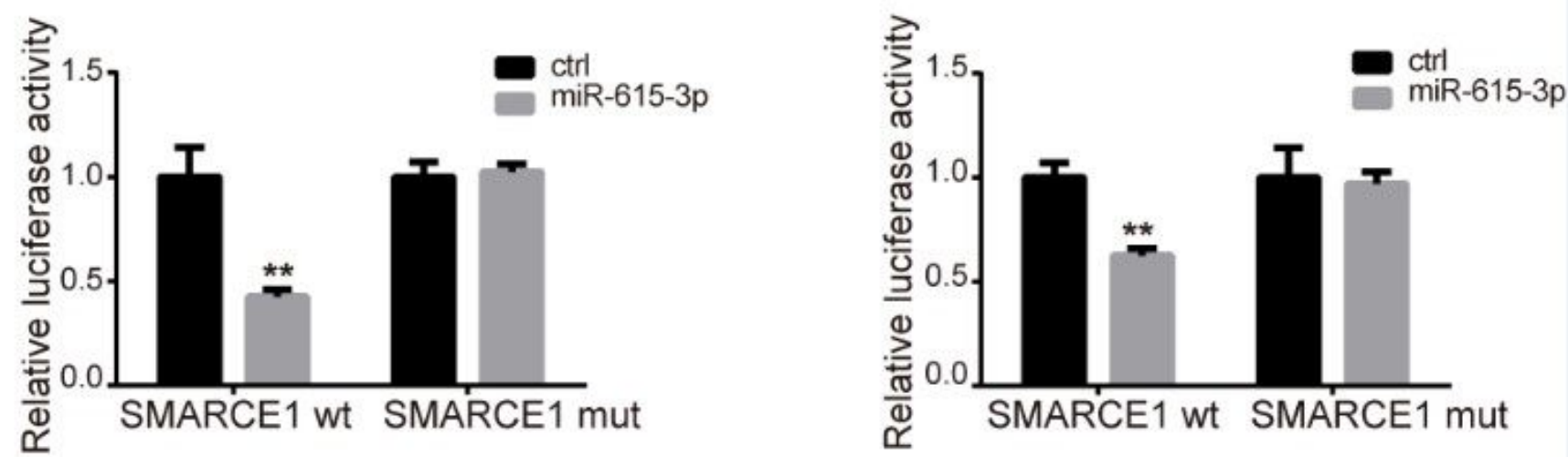

C
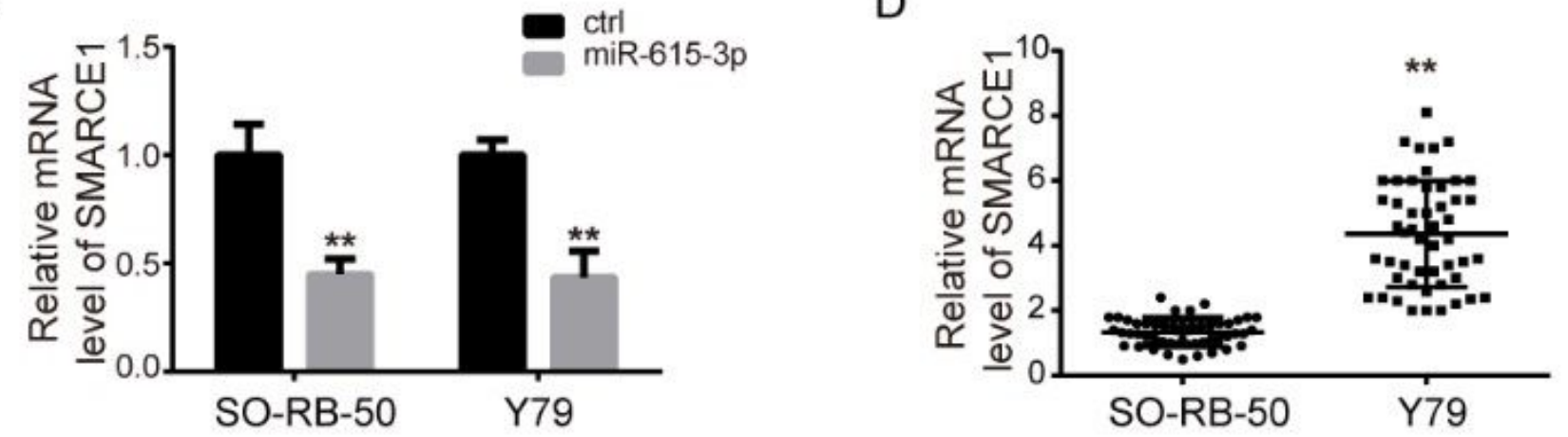

$\mathrm{E}$

$\mathrm{F}$
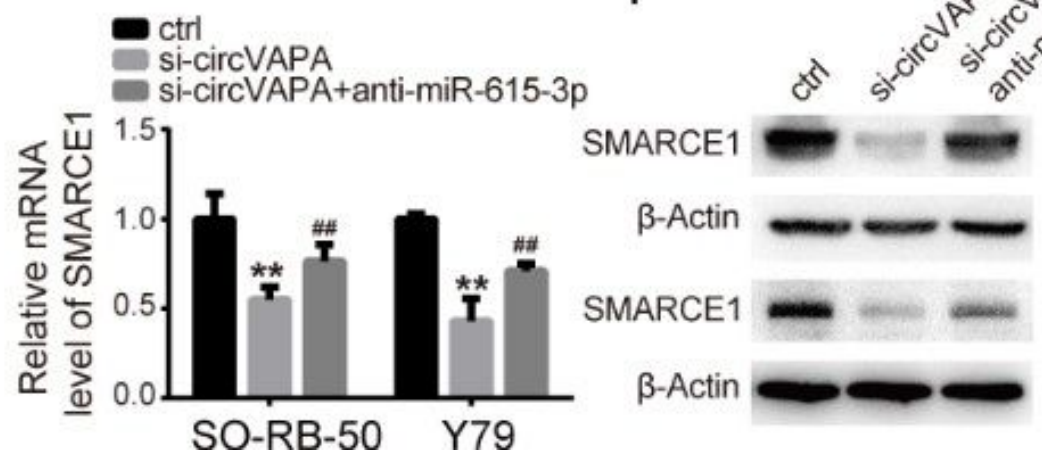

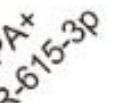

ctrl

si-circVAPA

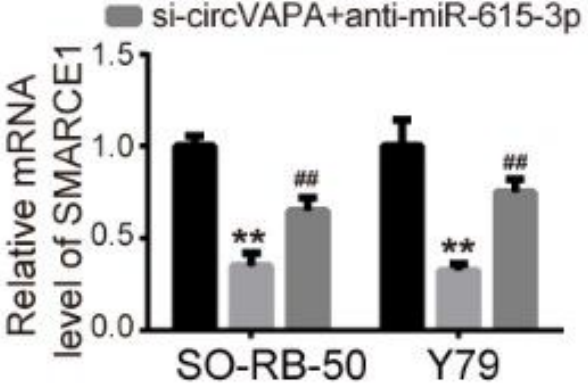

Figure 5

SMARCE1 was positively modulated by circVAPA via miR-615-3p. (A) Putative interacting sites predicted by TargetScan. (B) Luciferase activities were detected after co-transfection of miR-615-3p and SMARCE1 3'UTR wt/mut. (C) miR-615-3p was overexpressed, and miR-615-3p level was measured by qRT-PCR. (D) 
SMARCE1 expression levels in RB tissues and matched nearby normal ones were measured by RT-qPCR. (E-F) si-circVAPA was co-transfected with miR-615-3p inhibitor or negative control inhibitor, then SMARCE1 mRNA and protein levels were determined by qRT-PCR and western blot. ${ }^{*} \mathrm{P}<0.01$ compared with control group; \#\#P<0.01 compared with si-circVAPA group.

A

ctrl

sh-circVAPA

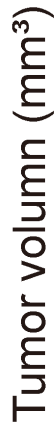

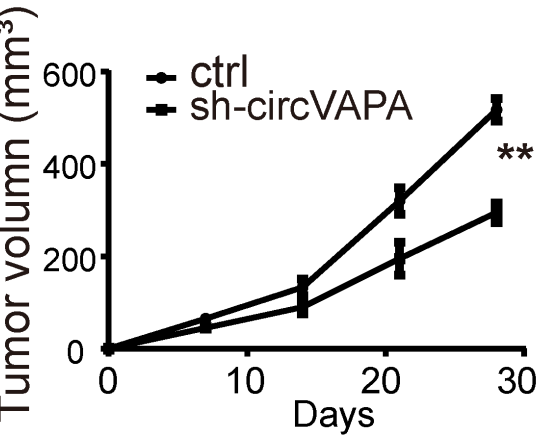

D

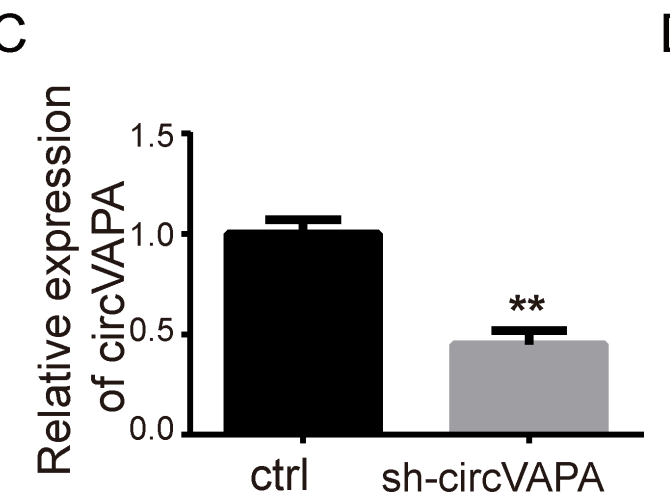

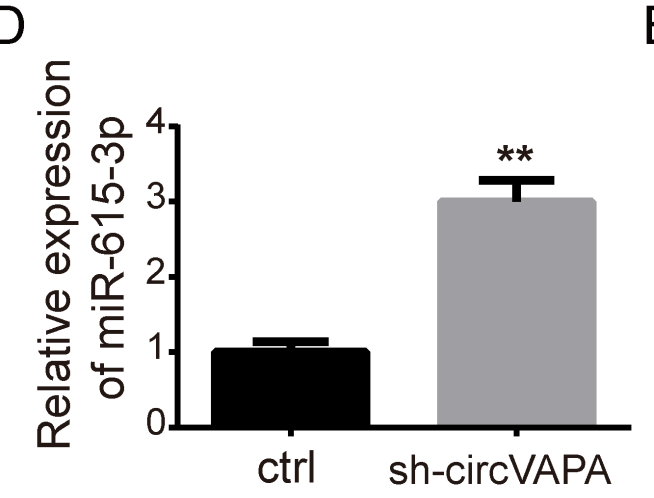

$\mathrm{B}$
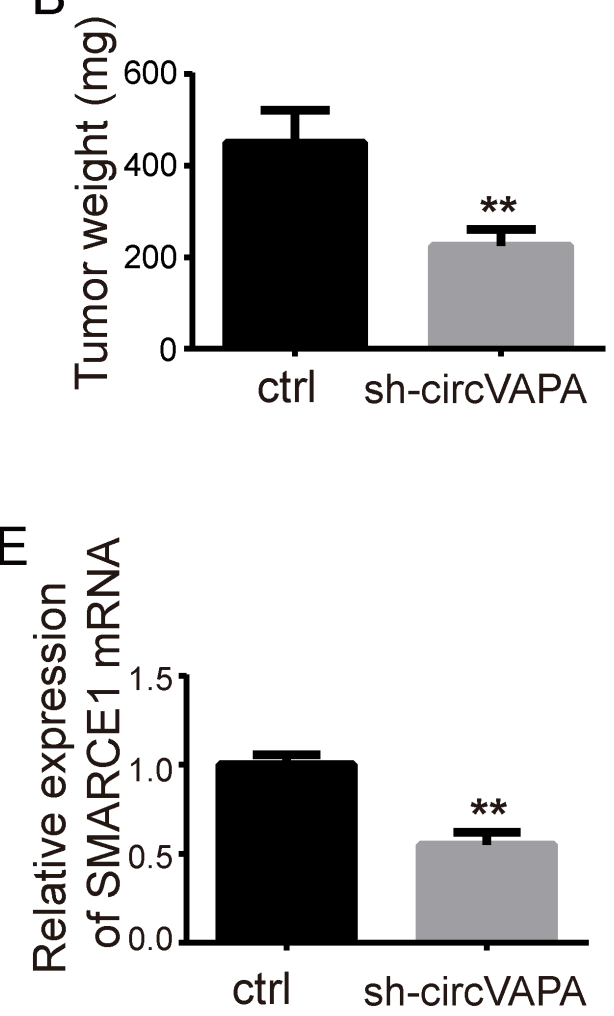

$\mathrm{F}$

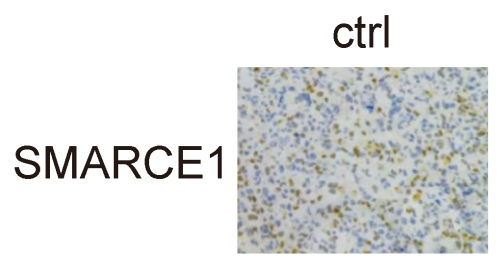

sh-circVAPA

G

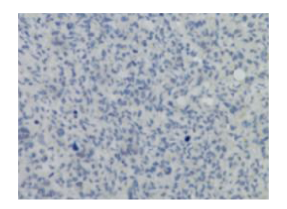

ctrl

sh-circVAPA

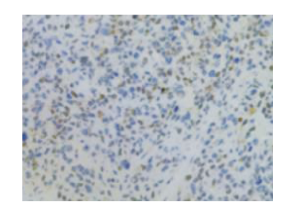

Figure 6

circVAPA silencing hampered RB tumorigenesis in mice. Mice were injected with $\mathrm{Y} 79$ cells expressing shcircVAPA or sh-scramble stably, (A-B) image of tumors removed 4 weeks later and the corresponding tumor sizes and weights; (C) circVAPA expression level in sh-circVAPA and sh-scramble mice; (D) miR615-3p expression levels in sh-circVAPA and sh-scramble mice; (E) SMARCE1 mRNA levels in sh-circVAPA and sh-scramble mice; (F) SMARCE1 expression levels were detected by $\mathrm{ICH}$; (G) Ki67 expression levels were assessed by $\mathrm{ICH} .{ }^{*} \mathrm{P}<0.01$. 